\title{
Targeted inhibition of dominant PI3-kinase catalytic isoforms increase expression of stem cell genes in glioblastoma cancer stem cell models
}

\author{
NICOLE M. JONES ${ }^{1}$, MATTHEW R. ROWE ${ }^{1}$, PETER R. SHEPHERD ${ }^{2,3}$ and MELANIE J. McCONNELL ${ }^{1,3,4}$ \\ ${ }^{1}$ School of Biological Sciences, Victoria University of Wellington, Wellington, New Zealand; \\ ${ }^{2}$ Department of Molecular Medicine and Pathology, University of Auckland, Auckland, New Zealand; \\ ${ }^{3}$ Maurice Wilkins Centre for Molecular Biodiscovery, Auckland; \\ ${ }^{4}$ Malaghan Institute of Medical Research, Wellington, New Zealand
}

Received January 12,2016; Accepted March 2, 2016

DOI: 10.3892/ijo.2016.3510

\begin{abstract}
Cancer stem cells (CSC) exhibit therapy resistance and drive self-renewal of the tumour, making cancer stem cells an important target for therapy. The PI3K signalling pathway has been the focus of considerable research effort, including in glioblastoma (GBM), a cancer that is notoriously resistant to conventional therapy. Different isoforms of the catalytic subunit have been associated with proliferation, migration and differentiation in stem cells and cancer stem cells. Blocking these processes in CSC would improve patient outcome. We examined the effect of isoform specific PI3K inhibitors in two models of GBM CSC, an established GBM stem cell line 08/04 and a neurosphere formation model. We identified the dominant catalytic PI3K isoform for each model, and inhibition of the dominant isoform blocked AKT phosphorylation, as did pan-PI3K/mTOR inhibition. Analysis of SOX2, OCT4 and MSII expression revealed that inhibition of the dominant p110 subunit increased expression of cancer stem cell genes, while pan-PI3K/mTOR inhibition caused a similar, though not identical, increase in cancer stem cell gene expression. This suggested that PI3K inhibition enhanced, rather than blocked, CSC activity. Careful analysis of the response to specific isoform inhibition will be necessary before specific subunit inhibitors can be successfully deployed against GBM CSC.
\end{abstract}

\section{Introduction}

Glioblastoma (glioblastoma multiforme, GBM) is a grade IV astrocytic tumour, the most aggressive form of astrocytic malignancy and the most common brain tumour in adults (1).

Correspondence to: Dr Melanie J. McConnell, School of Biological Sciences, Victoria University of Wellington, PO Box 600, Wellington 6140, New Zealand

E-mail: melanie.mcconnell@vuw.ac.nz

Key words: glioblastoma, cancer stem cell, PI3K, A66, TGX221, BEZ235
The disease can arise de novo, which accounts for $90 \%$ of cases, or as a secondary GBM, which is more common in younger people and progresses from a lower grade glial tumour. GBM is notoriously resistant to therapy, surgery cannot target the diffuse margins of the tumour, while cells have limited susceptibility to conventional radiation and temozolomide chemotherapy. With standard therapy, the median survival is 19 months post diagnosis (2). This dismal prognosis is in part due to the presence of cancer stem cells (CSC) in GBM. While the definition and characteristics of GBM CSC are controversial (3-6), these cells can generally considered to be intrinsically resistant to therapy (7), have self-renewal activity so can re-establish tumours after treatment (8) and are highly migratory and invasive (9), meaning they are likely to be present in the infiltrating edges of the tumour left behind after surgery. Therapies that target CSC and the characteristics of survival and self-renewal should dramatically improve the outcome for people with GBM.

Normal cellular processes of proliferation and survival are tightly regulated through a number of signalling pathways, including through the phosphatoinositide 3-kinase (PI3K) family proteins. The genes PIK3CA, PIK3CB, PIK3CD encode the catalytic subunit of class $\mathrm{I}_{\mathrm{A}}$ kinases $\mathrm{p} 110 \alpha, \mathrm{p} 110 \beta$ and $\mathrm{p} 110 \delta$, respectively, while the gene PIK3CG codes for a separate subunit class $\mathrm{I}_{\mathrm{B}}$ kinase $\mathrm{p} 110 \gamma$. Mutation and dysregulation of the PI3K/AKT/mTOR signalling axis is a major contributor to tumorigenic behaviour in cells, with key roles in proliferation, migration and epigenetic silencing of developmental pathways. Hyper-phosphorylation of AKT is frequent in cancers, particularly where PI3K activity is decoupled from EGF signal transduction through the loss of PTEN function (10). This increases proliferation and migration of cells $(11,12)$ particularly in glioblastoma, where $85 \%$ of tumours have activating mutations in the RTK/PI3K/AKT pathway (13). This has made the PI3K pathway a potential pharmacological target in glioblastoma treatment (14). Inhibition of various kinases within these pathways is an effective treatment for GBM in vitro, however, clinical translation of these findings remains to be clarified. This is due in part to cross-talk and plasticity between cell signalling pathways (15) and to the 
intrinsic resistance of GBM cells to apoptosis $(16,17)$. To overcome the adaptive response to PI3K/AKT/mTOR signalling, a complete understanding of the role of each individual effector is required.

The PI3K enzyme family mediate signal transduction via the phosphorylation of the 3'-OH of phosphatidylinositols localised to the internal surface of the cell membrane. Once activated, these messengers are responsible for downstream transduction through phosphorylation of AKT and the associated cellular responses. While class-I PI3K p110 isoforms share obvious structural similarities, a growing body of evidence describes discrete physiological roles for each isoform (18-24), with marked alterations in dominant isoform across malignancies (25), especially in PTEN deficient solid tumours (26). Activating point mutations are reported in the class $\mathrm{I}_{\mathrm{A}} \mathrm{PI} 3 \mathrm{~K}$ p110 $\alpha$ isoform in glioblastoma $(27,28)$, along with gene copy amplifications of both PI3K p110 $\alpha$ and PI3K p1108 $(29,30)$. Isoform specific inhibition may provide significant benefits if used in the appropriate genetic background (31). A number of strategies using isoform specific PI3K inhibitors, alone or in combination with additional compounds are currently in clinical trial $(26,32)$.

Most investigation has focused on PI3K activity in the proliferative and migratory phenotypes of differentiated glioblastoma cells $(14,25,33-36)$, however, little is known regarding the activity of PI3K isoforms in GBM CSC. In the present study, we examined expression and signalling of class $\mathrm{I}_{\mathrm{A}} \mathrm{PI} 3 \mathrm{~K}$ isoforms in two models of GBM CSC. The cell line 08/04 has high expression of embryonic and neural stem progenitor genes including SOX2, OCT4 and MSI1 and recapitulates a GBM phenotype following intra-cranial implantation (37). These cells were selected to model the effect of PI3K inhibition in maintenance of an established cancer stem cell phenotype. To determine how PI3K inhibition affects the acquisition of stem-like properties, an LN18 neurosphere model was utilised (37). PI3K isoforms p110 $\alpha, \mathrm{p} 110 \beta$ and $\mathrm{p} 110 \delta$ were selectively inhibited and effects on proliferation and migration assessed. This revealed a different dominant isoform in each model. Furthermore, changes in gene expression were evaluated following inhibition of the dominant isoform, to explore effects on stem-like and differentiation phenotypes. Inhibition increased transcription of cancer stem cell genes in both models, suggesting de-differentiation in response to blockade of the PI3K/AKT/mTOR signalling axis.

\section{Materials and methods}

Cell lines and tissue culture. The human GBM cell line LN18 was obtained from the American Type Culture Collection and was maintained in RPMI-1640 growth medium supplemented with $10 \%$ (v/v) fetal bovine serum (Life Technologies, Auckland, New Zealand). Cells were discarded within 20 passages of initial receipt and replaced with cryopreserved stock cultures. Primary human glioblastoma stem cells 08/04 (37) were maintained as an adherent monolayer in serum-free stem cell medium (SCM) supplemented with heparin, hEGF and bFGF (NeuroCult NS-A proliferation kit; Stem Cell Technologies, Tullamarine, VIC, Australia) as recommended by the manufacturer. All cultures were maintained at $37^{\circ} \mathrm{C}$ in a humidified $5 \% \mathrm{CO}_{2}$ atmosphere and subject to regular screening for myco- plasma contamination (e-Myco ${ }^{\mathrm{TM}}$ Mycoplasma PCR detection kit; Intron Biotechnology, Sangdaewon-dong, Korea).

PI3K inhibitors. A66 (38), TGX221 (39), IC87114 (40),

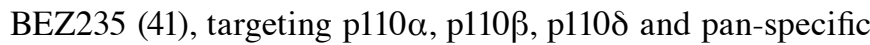
PI3K, respectively, were dissolved in sterile DMSO. The starting dose for each inhibitor was chosen based on the $\mathrm{IC}_{50}$ in a cell-free kinase assay, then titrated down from $100 \mathrm{x} \mathrm{IC}_{50}$ to find the maximal tolerated dose. A66 was used at $537.5 \mathrm{nM}$ $\left(12.5 \times \mathrm{IC}_{50}\right)$, TGX221 at $850 \mathrm{nM}\left(100 \mathrm{x} \mathrm{IC}{ }_{50}\right)$, IC87114 at $6 \mu \mathrm{M}$ $\left(100 \times \mathrm{IC}_{50}\right)$ and BEZ235 at $112.5 \mathrm{nM}\left(1.5 \mathrm{x} \mathrm{IC} \mathrm{I}_{50}\right)$.

$R T$ - $q P C R$. RNA was extracted using the ZR Quick-RNA MiniPrep kit (Zymo Research, Irvine, CA, USA) in accordance with the manufacturer's protocol. cDNA was synthesised from 250 ng of total RNA via the iScript cDNA synthesis kit (Bio-Rad Laboratories, Auckland, New Zealand). The reverse transcription was run with a Bio-Rad iCycle PCR machine with the following parameters: $25^{\circ} \mathrm{C} 5 \mathrm{~min}, 42^{\circ} \mathrm{C} 30 \mathrm{~min}$, $85^{\circ} \mathrm{C} 5 \mathrm{~min}$ and the sample was then held at $4^{\circ} \mathrm{C}$. QuantiTect Primer Assays (Qiagen, Melbourne, VIC, Australia) targeted to $18 \mathrm{~S}$ (QT00199367), HPRT1 (QT00059066), SOX-2 (QT00237601), MSI-1 (QT00025389), OCT-4 (QT00210840), GFAP (QT00081151), PIK3CA (QT00014861), PIK3CB (QT01668821) and PIK3CD (QT00091840) were used with SensiMix SYBR Low-ROX kit (Bioline, London, UK). The qPCR was run on the Applied Biosystems 7500 Real-Time PCR system (Applied Biosystems, Auckland, New Zealand) with the following parameters: Stage 1: $94^{\circ} \mathrm{C} 15 \mathrm{~min}$, Stage 2 (40 repeats): $94^{\circ} \mathrm{C} 15 \mathrm{sec}, 55^{\circ} \mathrm{C} 30 \mathrm{sec}, 72^{\circ} \mathrm{C} 35 \mathrm{sec}$. Each QuanTitect Primer Assay is pre-validated to have equivalent amplification efficiency, thus, direct normalisation to HPRT and $18 \mathrm{~s}$ was carried out via the $\Delta \Delta \mathrm{Ct}$ method. $\mathrm{Ct}$ (cycle threshold) values were exported from the 7500 System software into an Excel file (Microsoft, Redmond, WA, USA) and all following calculations were done using Excel software. The triplicate $\mathrm{Ct}$ values for each test primer pair were averaged and then normalized to the average $\mathrm{Ct}$ value of $18 \mathrm{~S}$ primer pair for each sample to give the $\Delta \mathrm{Ct}$ value. Then the difference between the $\Delta \mathrm{Ct}$ value for the control and $\Delta \mathrm{Ct}$ value for the treated cells was calculated to give a $\Delta \Delta \mathrm{Ct}$ value. The fold change in gene expression was calculated using $2^{-\Delta \Delta C t}$.

Growth factor signalling. Two million cells were plated in SCM (08/04) or complete RPMI-1640 (LN18) and allowed to proliferate for 2 days. Media were replaced with NeuroCult NS-A Medium with proliferation supplement and heparin, but without EGF or FGF (08/04), or RPMI-1640 without serum (LN18). After 16-h growth factor withdrawal, cells were treated with PI3K inhibitor or vehicle control for $1 \mathrm{~h}$ before addition of $20 \mathrm{ng} / \mathrm{ml} \mathrm{hEGF}$ and $10 \mathrm{ng} / \mathrm{ml} \mathrm{hFGF}-\beta(08 / 04)$ or $10 \%$ FBS v/v (LN18) to re-stimulate PI3K activity. Cells were incubated for $15 \mathrm{~min}$ then harvested for analysis by western blot analyis.

Western blotting. Cells were lysed in $70 \mathrm{mM} \mathrm{NaCl}, 20 \mathrm{mM}$ Tris, $0.1 \%$ Tween with $1 \mathrm{X}$ protease inhibitor (Complete ULTRA EDTA free; Roche, Auckland, New Zealand) and 1X phosphatase inhibitor (PhosStop; Roche) and maintained on ice for all subsequent steps. Soluble material was retained by centrifuga- 
tion of lysates and total protein quantified. Protein $(50 \mu \mathrm{g})$ was electrophoresed with Amersham GE Precast gels, transferred to PVDF membrane (Bio-Rad Laboratories) and blocked in 5\% BSA (ICP Bio, Auckland, New Zealand) in PBS. The primary antibodies used were: polyclonal rabbit anti-human pAKT Ser473, monoclonal rabbit anti-human pAKT Thr308 (C31E5E), polyclonal rabbit anti-human AKT (Cell Signaling Technology, Inc., Danvers, MA, USA) and monoclonal, mouse anti-human $\alpha$-tubulin (Sigma-Aldrich, Auckland, New Zealand). Primary antibodies were sequentially blotted at a concentration of 1:1,000 overnight followed by the appropriate anti-mouse or anti-rabbit horseradish peroxidase antibody at a concentration of 1:7,000 (Santa Cruz Biotechnology, Santa Cruz, CA, USA) and detected by enhanced chemiluminescence (SuperSignal Pico; Pierce, Auckland, New Zealand). Chemiluminescent images were captured by the Carestream Gel Logic 4000 Pro using Carestream MI NE software (Carestream, Rochester, NY, USA). Membranes were stripped (Restore Western Blot stripping buffer; Thermo Fisher Scientific, Auckland, New Zealand) and re-imaged between antibodies to confirm complete signal ablation.

MTS assay. 08/04 or LN18 cells were seeded at 25,000 cell/well on a 96-well plate in the appropriate complete growth media. Cells were allowed to establish for $24 \mathrm{~h}$ before addition of PI3K inhibitors, which were refreshed every $48 \mathrm{~h}$. Five days following treatment, $20 \mu \mathrm{l}$ of 3-(4,5-dimethylthiazol-2-yl)5-(3-carboxymethoxyphenyl)-2H-tetrazolium (MTS) reagent per well (CellTiter 96 AQueous One Solution; Promega, Madison, WI, USA) was added and incubated at $37^{\circ} \mathrm{C}, 5 \% \mathrm{CO}_{2}$ for $4 \mathrm{~h}$ before absorbance at $490 \mathrm{~nm}$ was measured. Data were normalised to the media only control.

Migration assay. 08/04 cells were seeded at 100,000 cells/well on a 24-well plate in SCM and allowed to become confluent (48-72 h), then pre-treated with PI3K inhibitor for $1 \mathrm{~h}$. A scratch injury was formed in the monolayer and photographed using the $\mathrm{x} 10$ objective lens every $2 \mathrm{~h}$ for a total of $16 \mathrm{~h}$ using the Olympus IX51 inverted microscope with ColorView III 5 MP camera and Cell A software (Olympus, Auckland, New Zealand). Cell migration was assessed at each time-point by area measurements generated in ImageJ software (33).

Differentiation assay. 08/04 cells were seeded in SCM at 96,000 cells/well on a 6 -well plate and allowed to establish for 2 days before addition of PI3K inhibitor, vehicle control, media only control or $10 \%$ FBS v/v to induce differentiation. Cells were incubated for 5 days with inhibitors renewed at day 3 , then harvested for RT-qPCR analysis of embryonic and neural stem cell genes.

Immunofluorescence of GFAP. 08/04 cells were seeded at 96,000 cells/well on coverslips in 6-well plates in SCM and treated for 5 days with PI3K inhibitor, vehicle control, media only control or $10 \% \mathrm{FBS}$ v/v to induce differentiation. Cells were fixed with $95 \%$ ethanol and $5 \%$ acetic acid v/v before permeabilisation with PBS/0.5\% Tween. Cells were blocked in $2 \%$ BSA in PBS with $0.1 \%$ Tween, probed with monoclonal mouse anti-GFAP (GA5; Cell Signaling Technology, Auckland, New Zealand) at $4^{\circ} \mathrm{C}$ overnight. Slides were washed
Table I. The PI3K catalytic subunits expressed in 08/04 cells.

\begin{tabular}{lccc}
\hline & $\alpha$ subunit & $\beta$ subunit & $\delta$ subunit \\
\hline Cycle threshold (Ct) & 27.04 & 28.86 & 34.04 \\
$\Delta \mathrm{Ct}(18 \mathrm{~s})$ & 12.86 & 14.69 & 19.86 \\
\hline
\end{tabular}

Expression of the PI3K catalytic subunits as shown by Cycle threshold $(\mathrm{Ct})$ and $\Delta \mathrm{Ct}$ (relative to $18 \mathrm{~s}$ ) values in $08 / 04$. Data are average of triplicate experiments.

then incubated with Alexa Fluor 488 goat anti-mouse IgG (Life Technologies, Auckland, New Zealand) at room temperature for $1 \mathrm{~h}$. Slides were washed, DAPI stained (UltraCruz mounting media; Santa Cruz Biotechnology) then imaged by the Olympus BX51TF fluorescent compound microscope (Olympus). Representative pictures of each condition were taken in brightfield, DAPI and UV channels and combined using Pixelmator software for Mac (Pixelmator, Vilnius, Lithuania).

Neurosphere induction. LN18 cells were pre-treated with inhibitor for 5 days in complete RPMI-1640, with drug refreshed at day 3. Cells were then seeded into 24-well plate format at 500 cells/well in SCM containing PI3K inhibitor. After 7 days in culture, developed neurospheres were harvested and prepared for analysis of embryonic and neural stem cell genes by RT-qPCR.

Statistical analysis of in vitro experiments. All statistical analyses were performed using GraphPad Prism version 4.00 (Graphpad Software, Inc., La Jolla, CA, USA). Student's unpaired, two-tailed t-tests were performed in sequence to assess statistical significance between two groups, with P-values of $<0.05$ considered as statistically significant.

\section{Results}

$P I 3 K$ pl10 $\alpha, p 110 \beta$ and $p 110 \delta$ are expressed but differentially active in 08/04 cells. To determine which isoforms of p110 were expressed in the GBM stem cell model 08/04, expression of $P I K 3 C A, P I K 3 C B$ and $P I K 3 C D$ was measured by quantitative RT-PCR. This confirmed that all isoforms were expressed, although PIK3CD transcript was present at a lower level than the other two (Table I).

To determine which isoform/s contributed to growth factor-driven signal transduction in the 0904-SC models, cells were pre-treated with a specific subunit inhibitor, either A66 $(\mathrm{p} 110 \alpha)$, TGX221 (p110ß) or IC87114 (p1108) and the effect on growth factor-driven signal transduction measured by phosphorylation of AKT (Fig. 1). The initial dose chosen was $100 \mathrm{x}$ the $\mathrm{IC}_{50}$ of each isoform in a cell-free kinase assay. For each inhibitor this was $10-20 \mathrm{x}$ the $\mathrm{IC}_{50}$ in cell-based assays, and each was well within the specific range (38-41). The pan-PI3K/mTOR inhibitor BEZ235 was used as a positive control for lost AKT phosphorylation, and to compare specific isoform inhibition to broad-spectrum inhibition. The BEZ235 dose was titrated from $100 \mathrm{x}$ to $1.5 \mathrm{x} \mathrm{IC}_{50}$. At this concentration, 

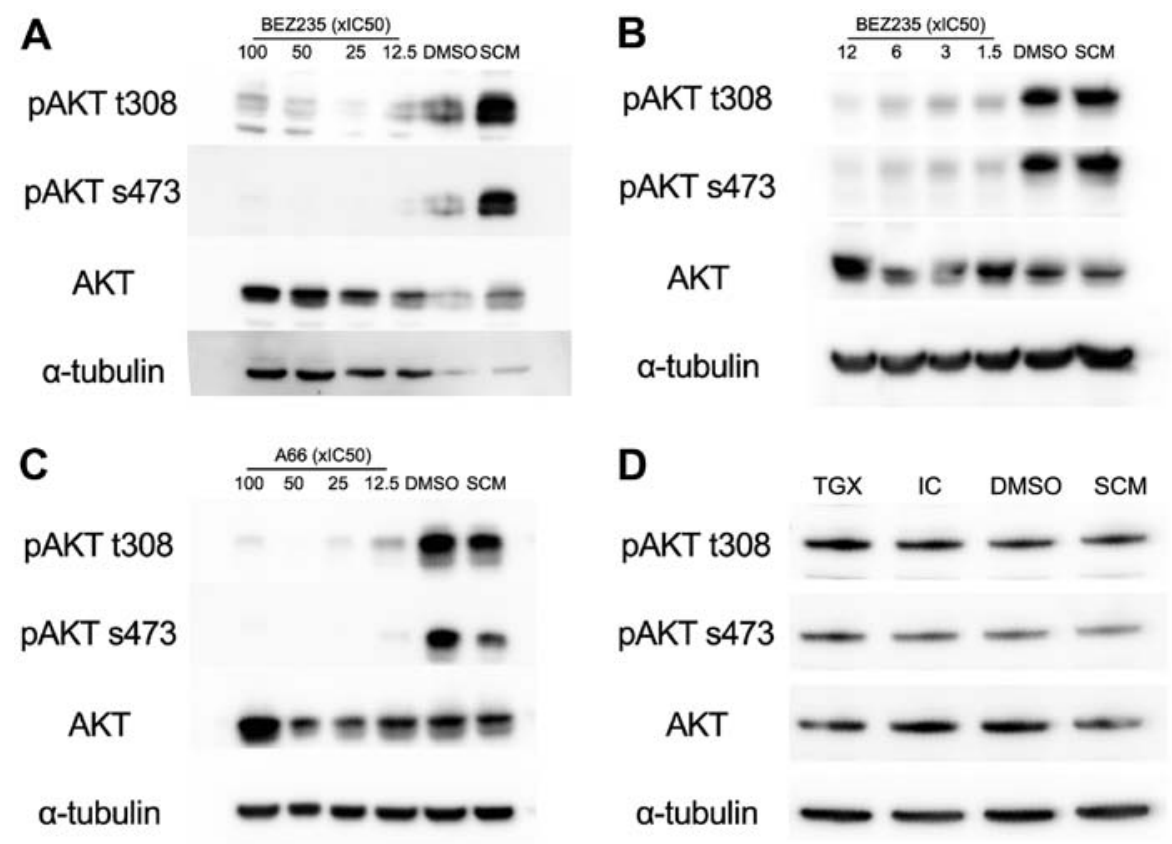

E MTS reduction by $08 / 04$ after drug treatment

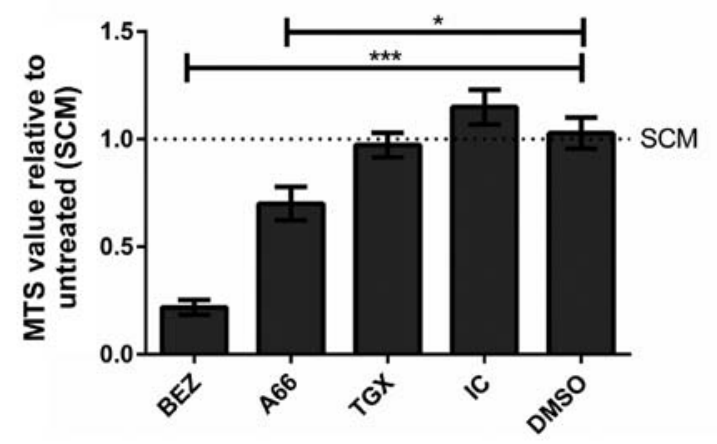

Figure 1. PI3K catalytic subunits are active in 08/04. Western blots showing a dose titration of (A) BEZ235 at 100-12.5x IC s0 , B) BEZ235 at 12-1.5x IC B $_{50}(\mathrm{C})$ A66 at 100-12.5x IC I $_{50}$ showing inhibition of pAKT at both $\mathrm{t} 308$ and s473 residues in 08/04, alongside DMSO and SCM (stem cell media) controls, representa-

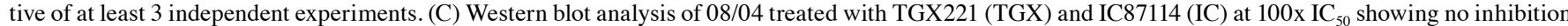
of pAKT, alongside DMSO and SCM controls. Data are representative of at least 3 independent experiments. (D) Graph showing reduction of MTS reagent by $08 / 04$ treated with inhibitors, relative to untreated controls. Columns represent mean values \pm SEM of triplicate experiments. ${ }^{*} \mathrm{P}<0.05,{ }^{* * *} \mathrm{P}<0.001$ Student's t-test.

substantial inhibition of phospho-AKT was observed (Fig. 1A and B), but cellular viability and processes were sufficiently conserved to purify intact RNA from treated cells. The dose of $112.5 \mathrm{nM}$ was used throughout. A similar titration of A66 was performed, and a dose of $12.5 \times \mathrm{IC}_{50}(537.5 \mathrm{nM})$ was identified. Inhibition of $\mathrm{p} 110 \alpha$ by $\mathrm{A} 66$ produced a significant reduction in AKT phosphorylation at all doses tested, with no detectable signal for serine 473 and a marked reduction of threonine 308 phosphorylation (Fig. 1C). In contrast, inhibition by TGX221 and IC87114 did not change phosphorylation at either residue at the highest starting dose (Fig. 1D) and had no observable impact on viability, indicating that PI3K isoforms p110 $\beta$ and p110 did not significantly contribute to signalling through AKT in the 08/04 model.

Inhibition of p110 $\alpha$, but not p110 $\beta$ or p110d, inhibits proliferation in 08/04 cells. To examine the functional consequence of selective PI3K p110 inhibition, cytoplasmic reduction of $\mathrm{NAD}(\mathrm{P}) \mathrm{H}$ was measured using MTS reduction $(42,43)$ following 5 days treatment with each PI3K inhibitor. Based on the dose titration, treatment was not accompanied by widespread cell death so the MTS value directly correlated with cell number, and changes in value reflected cellular proliferation. Consistent with the reduction of pAKT, inhibition of p1 $10 \alpha$ by A66, and PI3K/mTOR inhibition by BEZ235 significantly reduced final cell number, and hence proliferation, for 08/04 in contrast to untreated and vehicle treated cells (Fig. 1E). Neither TGX221 nor IC87114 affected final cell number, again consistent with the observation that $110 \beta$ and $110 \delta$ did not contribute to signalling through AKT in this model. These data were indicative of a cytostatic, rather than toxic, effect of inhibition, as observed previously in inhibition of the $\mathrm{PI} 3 \mathrm{~K} / \mathrm{AKT} / \mathrm{mTOR}$ axis $(15,44)$.

PI3K signalling does not drive migration in 08/04 cells. Given the potential for CSC to contribute to the invasive nature of glioblastoma in vivo, the effect of isoform selective PI3K inhibition on migration was assessed with the scratch assay. All 
A

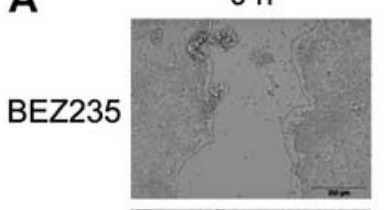

A66

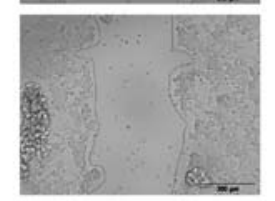

DMSO

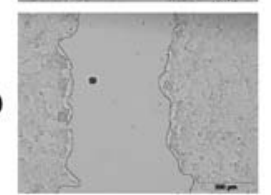

SCM

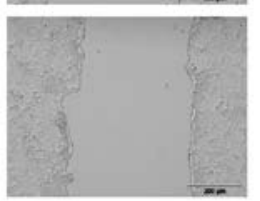

$12 \mathrm{~h}$
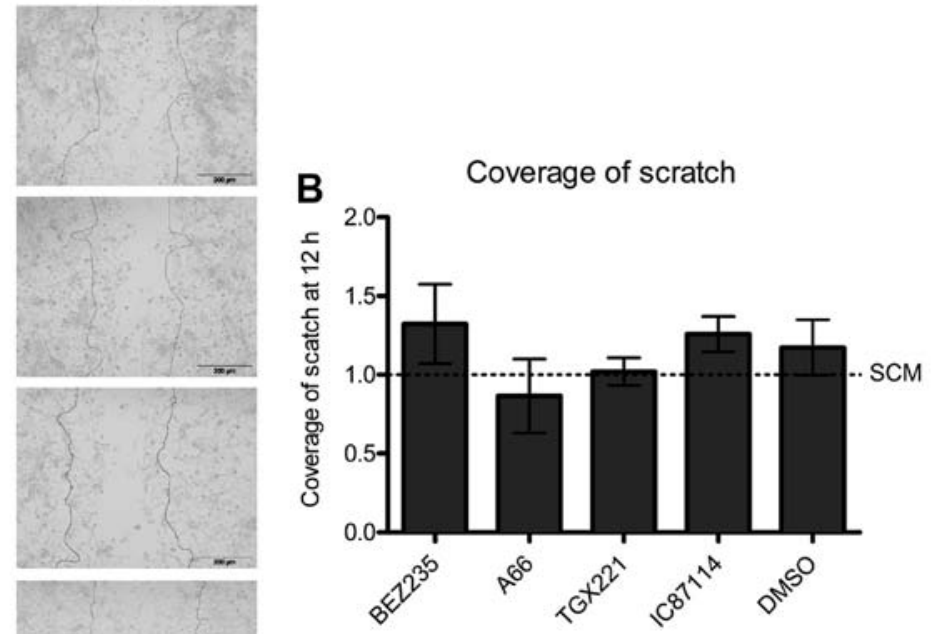

Figure 2. Migration of 08/04 cells was unchanged by inhibition with PI3K inhibitors. (A) Representative photos of scratch assay showing the migration of 08/04 pre-treated with PI3K inhibitors. (B) Percent coverage of scratch at $12 \mathrm{~h}$ for all treatment groups. Bars represent mean \pm SEM of triplicate experiments.

B
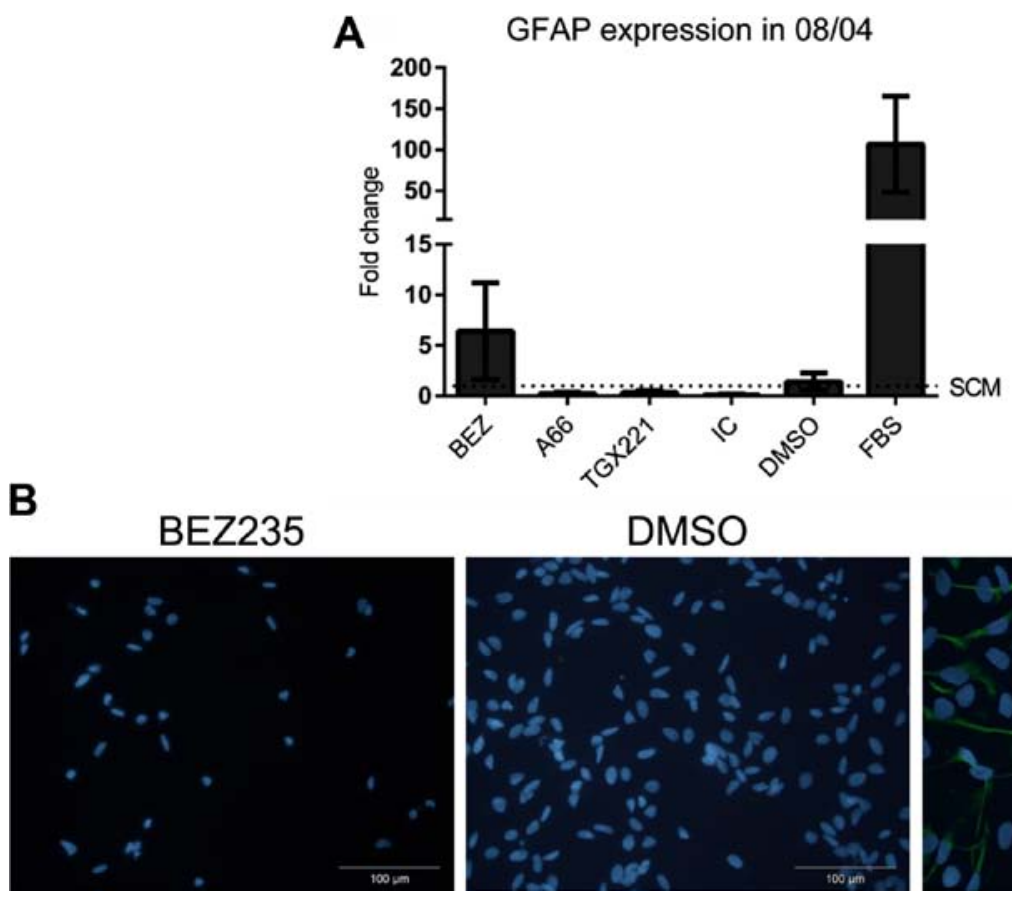

DMSO

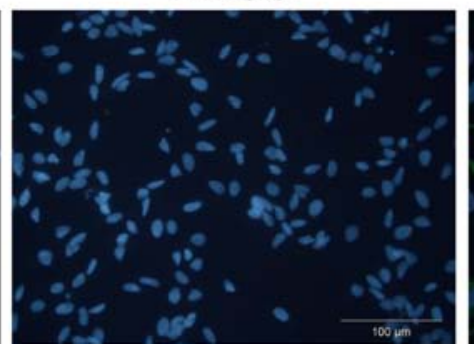

FBS

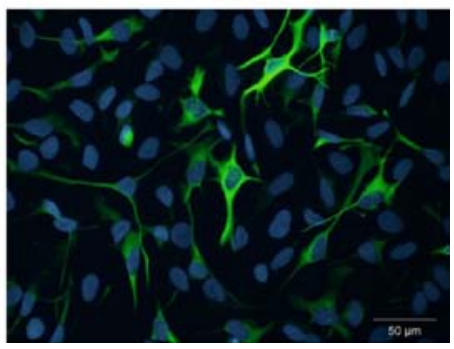

Figure 3. 08/04 exposed to PI3K inhibitors did not differentiate. (A) Expression of GFAP in 08/04 after exposure to PI3K inhibitors, DMSO control, SCM control and FBS containing media (FBS). Columns indicate mean \pm SEM of triplicate experiments. (B) Immunofluorescence of 08/04 indicating GFAP in green (Alexa Fluor-488) and nuclei in blue (DAPI) in BEZ235 treated cells, DMSO control and FBS. Data are representative of three independent experiments.

cells treated with PI3K inhibitors remained highly migratory, similar to vehicle treated cells (Fig. 2) and despite reduced AKT phosphorylation in the presence of A66 or BEZ235. No difference in migration was observed at any of multiple time-points, with all scratches filled in completely by $16 \mathrm{~h}$. Migration data from $12 \mathrm{~h}$ is shown.

PI3K inhibition does not induce differentiation of 08/04 cells. Inhibition of either $\mathrm{p} 110 \alpha$ or the PI3K/mTOR pathway suppressed proliferation of $08 / 04$ cells through a cytostatic mechanism, as no cell death was observed. We next determined the effect of isoform selective PI3K inhibition on differentiation of cells. GFAP is an intermediate filament protein of lineage committed glial cells, and expression indicates differentiation down the glial lineage. Using GFAP as a marker for differentiation, $08 / 04$ cells exposed to $10 \%$ FBS had 100 -fold upregulation of GFAP mRNA (Fig. 3A) and increased protein expression (Fig. 3B). However, isoform selective inhibition did not upregu- 


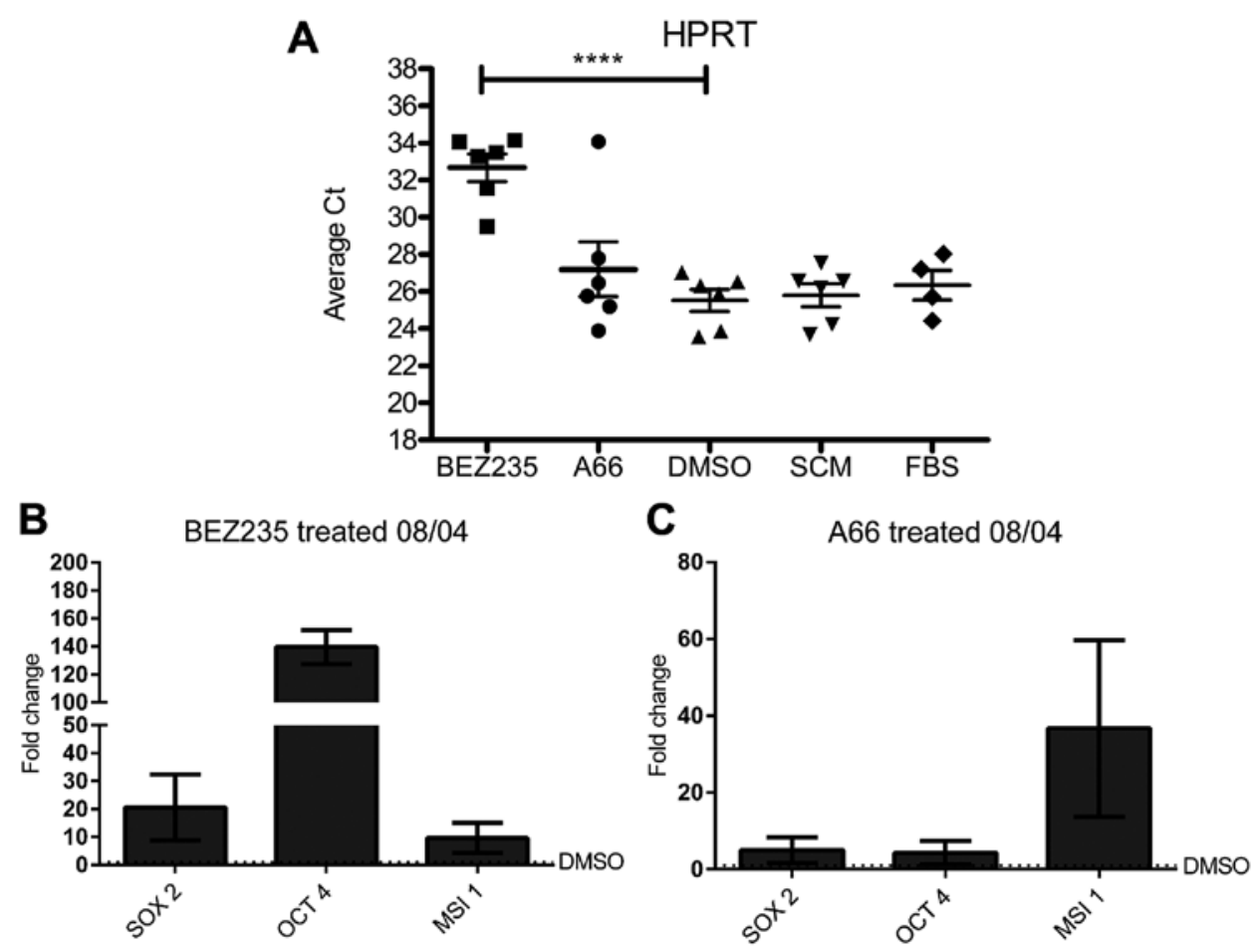

Figure 4. Treatment with PI3K inhibitors induced the expression of pluripotency and neural stem cell markers. (A) Cycle threshold (Ct) values for the expression of the housekeeping gene HPRT in 08/04 exposed to PI3K inhibitors BEZ235 (1.5x IC $\left.\mathrm{C}_{50}\right)$ and $\mathrm{A} 66\left(12.5 \mathrm{x} \mathrm{IC}_{50}\right)$, DMSO, SCM and FBS. Expression of pluripotency markers SOX 2 and OCT 4 and neural stem cell marker MSI 1 in 08/04 treated with: (B) BEZ235; and (C) A66. Columns represent mean \pm SEM of triplicate experiments. ${ }^{* * * *} \mathrm{P}<0.0001$ paired Student's t-test.

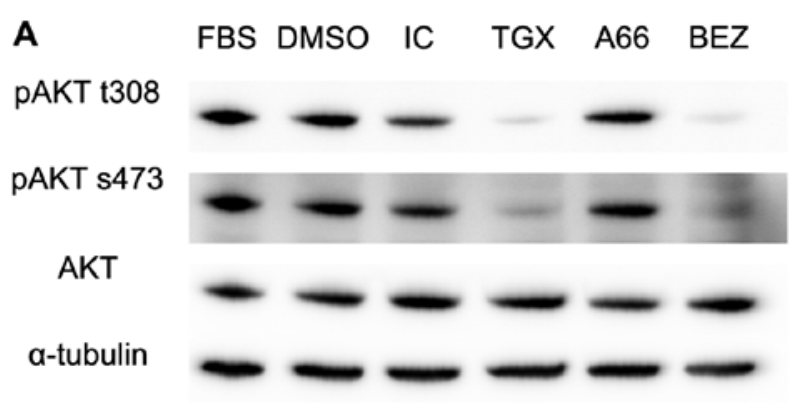

B MTS reduction by LN18 after drug treatment

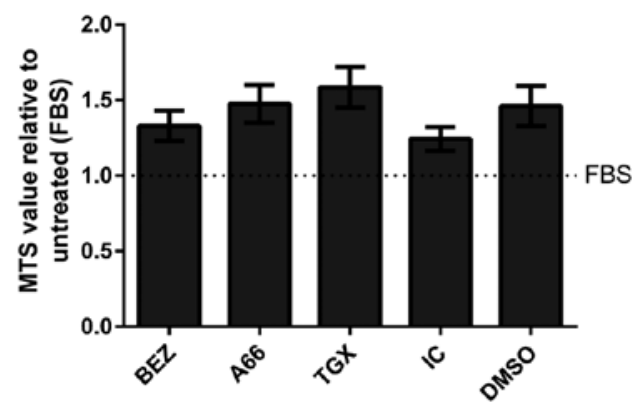

Figure 5. PI3K catalytic subunits were active in LN18. (A) Western blot analysis showing inhibition of pAKT with BEZ235 (1.5x IC 50 and TGX221 (100x $\left.\mathrm{IC}_{50}\right)$ at both $\mathrm{t} 308$ and $\mathrm{s} 473$ residues in LN18 alongside LN18 treated with A66 (12.5x $\left.\mathrm{IC}_{50}\right)$, IC87114 (100x IC s0 $_{0}$, DMSO and FBS (RPMI containg 10\% FBS) controls. Data are representative of at least 3 independent experiments. (B) Graph showing reduction of MTS reagent by LN18 treated with inhibitors, relative to untreated controls. Columns represent mean values \pm SEM of triplicate experiments.

late the GFAP transcript (Fig. 3A) and neither BEZ235 nor A66 induced expression of GFAP protein (Fig. 3B). This suggested that PI3K inhibition was not associated with increased differentiation of glioblastoma cancer stem cells.

Surprisingly, inhibition of PI3K by BEZ235 reproducibly appeared to upregulate GFAP mRNA, in contrast to the lack of GFAP protein detected by immunofluorescence. On further investigation, treatment by BEZ235 was found to decrease the relative transcript abundance of the gene used for normalisation of gene expression, HPRT (Fig. 4A) as well as a number of other genes commonly used for normalisation (18s rRNA, TBP, $\beta$-actin; data not shown). This led to the apparent increase in GFAP transcription. Importantly, the effect was restricted to BEZ235 treatment (Fig. 4A).
This prevented direct comparison of gene expression between BEZ235 treated cells and other inhibitor treatments. However, within a treatment group, gene expression data were directly comparable, and this approach was used for all further qRT-PCR analysis.

PI3K inhibition increased expression of CSC genes in 08/04 cells. We next examined expression of stem cell genes in our GBM CSC model. Cells were treated with inhibitor for 5 days and expression of the embryonic and neural stem cell transcription factors SOX2, OCT4 and MSI1 was measured. A surprising but consistent pattern of upregulation of CSC gene expression was observed. When compared to vehicle treated cells, A66 treatment led to an average 5-fold increase in SOX2 
A

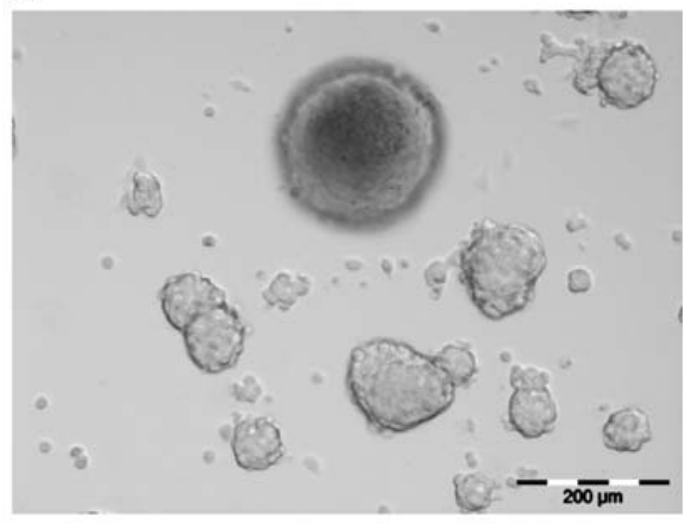

C

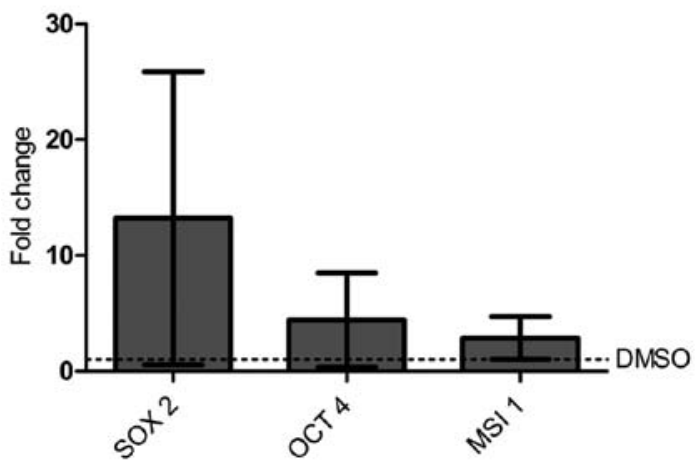

B

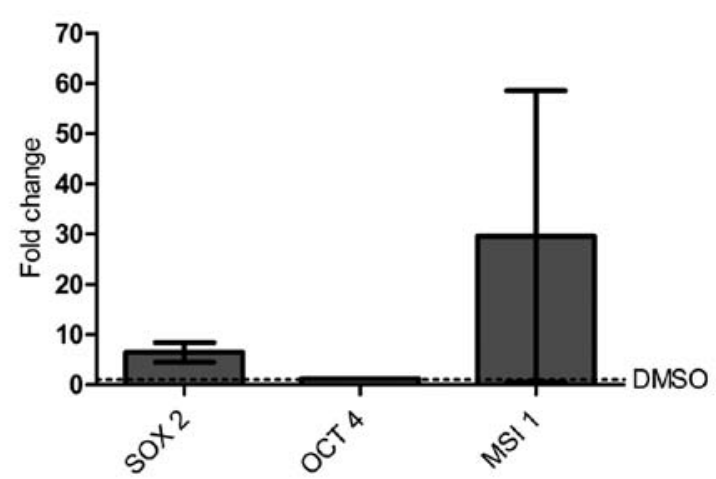

D

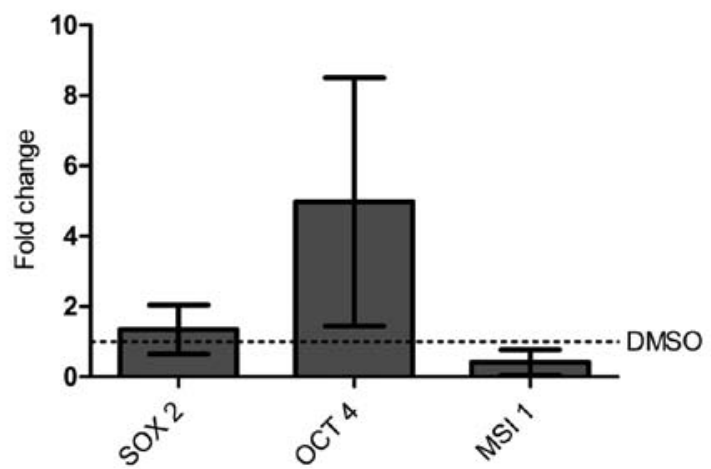

Figure 6. Induction of stem cell gene expression is changed by PI3K inhibition. (A) Representative picture of spheres formed when LN18 cells were grown in SCM. Expression of self-renewal marker SOX2, pluripotency marker OCT4 and neural stem cell marker MSI1 in LN18 cells in: (B) untreated cells grown in SCM; (C) cell treated with BEZ235, then grown in SCM; (D) cells treated with TGX221, then grown in SCM. Columns indicate mean \pm SEM of duplicate experiments.

Table II. The PI3K catalytic subunits expressed in LN18.

\begin{tabular}{lrcr}
\hline & $\alpha$ subunit & $\beta$ subunit & $\delta$ subunit \\
\hline Cycle threshold $(\mathrm{Ct})$ & 27.98 & 29.65 & 29.55 \\
$\Delta \mathrm{Ct}(\mathrm{HPRT})$ & 1.81 & 3.91 & 4.53
\end{tabular}

Expression of the PI3K catalytic subunits as shown by cycle threshold $(\mathrm{Ct})$ and $\Delta \mathrm{Ct}$ (relative to HPRT) values in LN18. Data are average of triplicate experiments.

and OCT4, and a 30-fold average increase in MSI1 mRNA (Fig. 4C). Treatment with BEZ235 also increased expression of SOX2 and MSI1, with a notable increase in OCT4 expression (Fig. 4B).

PI3K p110 $\alpha, p 110 \beta$ and $p 110 \delta$ are expressed but differentially active in LN18. The 08/04 cells effectively model maintenance of an established cancer stem cell phenotype. In order to determine the effect of PI3K p110 inhibition on the acquisition of CSC characteristics, a neurosphere formation model was used. LN18 cells proliferate as an adherent monolayer when cultured in $10 \% \mathrm{FBS}$, but form neurospheres on transfer to neural stem cell media at low cell density (37). This acquisition of a stem cell phenotype, or de-differentiation, is a model of cancer stem cell initiation.
Basal expression of PI3K p110 $\alpha$, p110 $\beta$ and p110 in serumgrown GBM cell line LN18 was established by qRT-PCR and the isoforms found to be of equal abundance (Table II). Analysis of signalling through the PI3K/AKT/mTOR axis revealed a difference in the dominant isoform between LN18 and $08 / 04$. In contrast to $08 / 04$, inhibition of catalytic subunit p110 $\alpha$ by A66 had no effect on pAKT relative to vehicle control (Fig. 5A). Instead, inhibition of p110 $\beta$ by TGX221 suppressed phosphorylation at both sites. Inhibition of p1108 by IC87114 had no significant effect on the phosphorylation of AKT at either residue, similar to 08/04. As expected, inhibition of PI3K/mTOR activity by BEZ235 supressed phosphorylation of AKT at both threonine 308 and serine 473. Despite suppressed AKT phosphorylation by BEZ235 and TGX221, there was no significant difference in final number of LN18 cells (Fig. 5B) following PI3K inhibition, implying no loss of proliferation or increase in cell death.

PI3K inhibition prior to neurosphere formation enhances expression of neural and embryonic transcription factors. Formation of LN18 neurospheres was previously characterised by upregulation of embryonic and neural stem cell gene transcription (37). LN18 cells were pre-treated with TGX221, BEZ235 or DMSO, then transferred to serum-free stem cell media to induce sphere formation (Fig. 6A). The effect of sphere formation on expression of the CSC gene panel was compared between DMSO and inhibitor treated cells, either 
BEZ235 (Fig. 6C) or TGX221 (Fig. 6D). There was no significant change in the rate of sphere formation (data not shown), but in each case, expression of at least 1 stem cell gene increased with PI3K inhibition, with a notable increase in OCT4 expression compared to untreated cells, similar to the $08 / 04$ data (Fig. 4B). Also similar to PI3K inhibition of $08 / 04$, increased gene expression was more pronounced with pan-PI3K/mTOR inhibition (Fig. 6C), again suggesting that multiple kinases regulated CSC gene expression.

\section{Discussion}

Activation of the PI3K/AKT/mTOR signalling pathway reportedly enriches for highly tumorigenic stem-like cancer cells $(45,46)$ and PI3K inhibition promotes differentiation, potentially reducing the self-renewal potential of cancer stem cells within tumours. The different isoforms of the PI3K catalytic subunit, $\mathrm{p} 110 \alpha, \beta$ and $\delta$, have been suggested to have differential roles in pathway activation, and differential effects on proliferation, migration and differentiation. Based on the de-differentiated, self-renewing nature of glioblastoma cancer stem cells, we hypothesised that isoform-specific PI3K inhibition might specifically target components of cancer stem cell activity as has been found for embryonic stem cells (47). We looked at activity of the PI3K catalytic subunits in two different GBM cancer stem cell models, using specific inhibitors to look first at any role in cancer stem cell proliferation, and secondly in the acquisition of a stem-like phenotype. The origin of the GBM CSC is generally thought to be the acquisition of key stem cell characteristics by a lineage-committed cell (48). The LN18 sphere formation assay was used to look specifically at PI3K activity in the acquisition of stem cell gene expression, as a marker of de-differentiation of a lineage-committed cell.

The specificity and selectivity of the PI3K inhibitors was critical to these experiments. The dose titrations started at $100 \mathrm{x}$ the $\mathrm{IC}_{50}$ for a cell-free kinase assay. At this dose, there was no effect of p1108 inhibition in either cell line with IC87114, which we interpreted as no activity. This was equivalent to $12 \mathrm{x}$ the cellular $\mathrm{IC}_{50}$. Similarly, there was no effect of TGX221 on $08 / 04$, at $17 \mathrm{x}$ the cellular $\mathrm{IC}_{50}$. While we have not formally excluded that either inhibitor would not have been effective at higher concentration this seems unlikely. There is also the possibility of non-specific activity if an inhibitor is used at a high dose. There was no non-specific activity of TGX221 and IC87114, but this could potentially have been observed with the p110 $\alpha$ inhibitor. However, selectivity of A66 is very high. At $537.5 \mathrm{nM}$, the dose used in this study, there is $>40$-fold higher concentration needed to inhibit the $\delta$ isoform, and $>400$-fold for inhibition of $\beta$ (38). Hence, we were well within the selective range.

While activation of the overall PI3K pathway led to constitutive AKT phosphorylation in both GBM CSC models, the activity of the p110 catalytic sub-units were different in each model. A single dominant isoform was identified in each model, which differed between the two models, p110 $\alpha$ was active in $08 / 04$, and p110 $\beta$ in the LN18 neurosphere model. Inhibition of PI3K, whether specific to the dominant p110 isoform, or pan-PI3K/mTOR inhibition, had similar effects on proliferation in each model. PI3K inhibition blocked AKT phosphorylation and reduced proliferation in 08/04. However, neither inhibitor had any effect on LN18 proliferation despite blocking AKT phosphorylation. The two GBM cell lines used are very similar in appearance and behaviour (37), but these data indicate that there are fundamental differences underlying their PI3K biology. This could include differences in the mutational status of the PI3K pathway, such as PTEN loss of function or activating PI3K mutations. In this regard, LN18 cells are known to have wild-type PTEN (49), but the genetic background of the 08/04 line is completely unknown. As differential expression of targets obviously leads to differential efficacy of targeted therapies, these data highlight the importance of further study into the PI3K pathway in GBM before any successful implementation of PI3K inhibition for cancer stem cell targeting.

A recently proposed competition model for p110 association with activated receptor tyrosine kinases (RTKs) (18) highlights the differential role of the isoforms in the regulation of PI3K activity. Differences in both lipid- and protein-kinase activities have been described for PI3K p110 isoforms $(50,51)$, which allow precise control over downstream transduction in response to activating signals. In addition, rebound signalling in other pathways following inhibition of dominant signalling isoforms has been identified in various cell lines $(52,53)$, demonstrating the adaptive nature of cancer cell signalling pathways and consequent cell survival.

Inhibition of either PI3K/mTOR or the dominant isoform altered expression of embryonic and neural stem cell genes in both CSC models, with overall increased stem cell gene expression. The precise effect of PI3K inhibition on stem cell gene expression varied between inhibitors. Whether this difference between pan-PI3K/mTOR and dominant isoform inhibition indicates a link between a given isoform and a corresponding specific CSC gene is not clear. Any contributory role of minor p110 isoforms, or of other signalling pathways to CSC gene expression requires further analysis.

Given that PI3K/AKT activation was previously reported to increase cancer stem cell gene expression $(45,46)$, the increased stem cell gene expression with PI3K inhibition was unexpected and inconsistent with those previous reports. Instead, it reflected data on PI3K inhibition in embryonic stem cells (47). It is possible that in our models, PI3K inhibition directly increased cancer stem cell gene expression, and simply reflected de-differentiation and acquisition of a more stem-like phenotype. Alternatively, the increase we observed could result from a more complex disruption to the stem cell phenotype. We speculate that while PI3K inhibition attempted to force cells to differentiate, another signalling pathway intervened to push stem gene expression back and restore the CSC phenotype, limiting the effect. In this circumstance, the increased stem cell gene expression we observed could be an 'over-shoot' phenomenon. Signal transduction in the PI3K/AKT/mTOR axis is partially mediated through a cross-inhibitory relationship with the MEK/ERK pathway in GBM (54). MEK/ERK signalling is highly integrated with the downstream transcriptional activation of embryonic and neural stem cell genes (55) and it is plausible that PI3K/MEK/ ERK cross-talk plays a role in cancer stem cell gene expression and activity $(56,57)$.

Regardless of the mechanism, increased expression of genes that drive a cancer stem cell phenotype would make 
PI3K inhibition counter-productive for cancer stem cell targeting, and for treatment of GBM. These data illustrate the complexity of PI3K biology in the cancer stem cell phenotype. A careful analysis of the role of other signalling pathways in the response to specific isoform inhibition will be necessary before these inhibitors can be successfully deployed against GBM cancer stem cells, or other tumours with a cancer stem cell component.

\section{References}

1. Louis DN, Ohgaki H, Wiestler OD, Cavenee WK, Burger PC, Jouvet A, Scheithauer BW and Kleihues P: The 2007 WHO classification of tumours of the central nervous system. Acta Neuropathol 114: 97-109, 2007.

2. Stupp R, Hegi ME, Mason WP, van den Bent MJ, Taphoorn MJ, Janzer RC, Ludwin SK, Allgeier A, Fisher B, Belanger K, et al; European Organisation for Research and Treatment of Cancer Brain Tumour and Radiation Oncology Groups; National Cancer Institute of Canada Clinical Trials Group: Effects of radiotherapy with concomitant and adjuvant temozolomide versus radiotherapy alone on survival in glioblastoma in a randomised phase III study: 5-year analysis of the EORTC-NCIC trial. Lancet Oncol 10: 459-466, 2009.

3. Maugeri-Saccà M, Di Martino S and De Maria R: Biological and clinical implications of cancer stem cells in primary brain tumors. Front Oncol 3: 6, 2013.

4. Cruceru ML, Neagu M, Demoulin JB and Constantinescu SN Therapy targets in glioblastoma and cancer stem cells: Lessons from haematopoietic neoplasms. J Cell Mol Med 17: 1218-1235, 2013.

5. Lathia JD: Cancer stem cells: Moving past the controversy. CNS Oncol 2: 465-467, 2013.

6. Hale JS, Sinyuk M, Rich JN and Lathia JD: Decoding the cancer stem cell hypothesis in glioblastoma. CNS Oncol 2: 319-330, 2013.

7. Beier D, Schulz JB and Beier CP: Chemoresistance of glioblastoma cancer stem cells - much more complex than expected. Mol Cancer 10: 128, 2011

8. Yan K, Yang K and Rich JN: The evolving landscape of glioblastoma stem cells. Curr Opin Neurol 26: 701-707, 2013.

9. Molina JR, Hayashi Y, Stephens C and Georgescu MM: Invasive glioblastoma cells acquire stemness and increased Akt activation. Neoplasia 12: 453-463, 2010.

10. Cantley LC and Neel BG: New insights into tumor suppression: PTEN suppresses tumor formation by restraining the phosphoinositide 3-kinase/AKT pathway. Proc Natl Acad Sci USA 96: 4240-4245, 1999.

11. Kwiatkowska A, Kijewska M, Lipko M, Hibner U and Kaminska B: Downregulation of Akt and FAK phosphorylation reduces invasion of glioblastoma cells by impairment of MT1-MMP shuttling to lamellipodia and downregulates MMPs expression. Biochim Biophys Acta 1813: 655-667, 2011.

12. Nakada M, Nakada S, Demuth T, Tran NL, Hoelzinger DB and Berens ME: Molecular targets of glioma invasion. Cell Mol Life Sci 64: 458-478, 2007.

13. Cancer Genome Atlas Research Network: Comprehensive genomic characterization defines human glioblastoma genes and core pathways. Nature 455: 1061-1068, 2008.

14. Westhoff MA, Karpel-Massler G, Brühl O, Enzenmüller S, La Ferla-Brühl K, Siegelin MD, Nonnenmacher L and Debatin KM: A critical evaluation of PI3K inhibition in Glioblastoma and Neuroblastoma therapy. Mol Cell Ther 2: 32, 2014.

15. Fan QW, Knight ZA, Goldenberg DD, Yu W, Mostov KE, Stokoe D, Shokat KM and Weiss WA: A dual PI3 kinase/mTOR inhibitor reveals emergent efficacy in glioma. Cancer Cell 9: 341-349, 2006.

16. Lefranc F, Brotchi J and Kiss R: Possible future issues in the treatment of glioblastomas: Special emphasis on cell migration and the resistance of migrating glioblastoma cells to apoptosis. J Clin Oncol 23: 2411-2422, 2005.

17. Stegh AH, Chin L, Louis DN and DePinho RA: What drives intense apoptosis resistance and propensity for necrosis in glioblastoma? A role for Bcl2L12 as a multifunctional cell death regulator. Cell Cycle 7: 2833-2839, 2008.
18. Utermark T, Rao T, Cheng H, Wang Q, Lee SH, Wang ZC, Iglehart JD, Roberts TM, Muller WJ and Zhao JJ: The p110 $\alpha$ and p1 $10 \beta$ isoforms of PI3K play divergent roles in mammary gland development and tumorigenesis. Genes Dev 26: 1573-1586, 2012.

19. Knight ZA, Gonzalez B, Feldman ME, Zunder ER, Goldenberg DD, Williams O, Loewith R, Stokoe D, Balla A, Toth B, et al: A pharmacological map of the PI3-K family defines a role for p110alpha in insulin signaling. Cell 125: 733-747, 2006.

20. Chaussade C, Rewcastle GW, Kendall JD, Denny WA, Cho K, Grønning LM, Chong ML, Anagnostou SH, Jackson SP, Daniele N, et al: Evidence for functional redundancy of class IA PI3K isoforms in insulin signalling. Biochem J 404: 449-458, 2007.

21. Zhao JJ, Cheng H, Jia S, Wang L, Gjoerup OV, Mikami A and Roberts TM: The p110alpha isoform of PI3K is essential for proper growth factor signaling and oncogenic transformation. Proc Natl Acad Sci USA 103: 16296-16300, 2006.

22. Foukas LC, Claret M, Pearce W, Okkenhaug K, Meek S, Peskett E, Sancho S, Smith AJ, Withers DJ and Vanhaesebroeck B: Critical role for the p110alpha phosphoinositide-3-OH kinase in growth and metabolic regulation. Nature 441: 366-370, 2006.

23. Graupera M, Guillermet-Guibert J, Foukas LC, Phng LK, Cain RJ, Salpekar A, Pearce W, Meek S, Millan J, Cutillas PR, et al: Angiogenesis selectively requires the p110alpha isoform of PI3K to control endothelial cell migration. Nature 453: 662-666, 2008.

24. Okkenhaug K, Turner M and Gold MR: PI3K signaling in B cell and T cell biology. Front Immunol 5: 557, 2014.

25. Schmit F, Utermark T, Zhang S, Wang Q, Von T, Roberts TM and Zhao JJ: PI3K isoform dependence of PTEN-deficient tumors can be altered by the genetic context. Proc Natl Acad Sci USA 111: 6395-6400, 2014.

26. Thorpe LM, Yuzugullu H and Zhao JJ: PI3K in cancer: Divergent roles of isoforms, modes of activation and therapeutic targeting. Nat Rev Cancer 15: 7-24, 2015.

27. Gallia GL, Rand V, Siu IM, Eberhart CG, James CD, Marie SK, Oba-Shinjo SM, Carlotti CG, Caballero OL, Simpson AJ, et al: PIK3CA gene mutations in pediatric and adult glioblastoma multiforme. Mol Cancer Res 4: 709-714, 2006.

28. Samuels Y, Wang Z, Bardelli A, Silliman N, Ptak J, Szabo S, Yan H, Gazdar A, Powell SM, Riggins GJ, et al: High frequency of mutations of the PIK3CA gene in human cancers. Science 304: $554,2004$.

29. Mizoguchi M, Nutt CL, Mohapatra G and Louis DN: Genetic alterations of phosphoinositide 3-kinase subunit genes in human glioblastomas. Brain Pathol 14: 372-377, 2004.

30. Hui AB, Lo KW, Yin XL, Poon WS and Ng HK: Detection of multiple gene amplifications in glioblastoma multiforme using array-based comparative genomic hybridization. Lab Invest 81: 717-723, 2001.

31. Jia S, Roberts TM and Zhao JJ: Should individual PI3 kinase isoforms be targeted in cancer? Curr Opin Cell Biol 21: 199-208, 2009.

32. Fruman DA and Rommel C: PI3K and cancer: Lessons, challenges and opportunities. Nat Rev Drug Discov 13: 140-156, 2014.

33. Filbin MG, Dabral SK, Pazyra-Murphy MF, Ramkissoon S, Kung AL, Pak E, Chung J, Theisen MA, Sun Y, Franchetti Y, et al: Coordinate activation of Shh and PI3K signaling in PTENdeficient glioblastoma: New therapeutic opportunities. Nat Med 19: 1518-1523, 2013.

34. Jhanwar-Uniyal M, Albert L, McKenna E, Karsy M, Rajdev P, Braun A and Murali R: Deciphering the signaling pathways of cancer stem cells of glioblastoma multiforme: Role of Akt/ mTOR and MAPK pathways. Adv Enzyme Regul 51: 164-170, 2011.

35. Paul-Samojedny M, Pudełko A, Suchanek-Raif R, Kowalczyk M, Fila-Daniłow A, Borkowska P and Kowalski J: Knockdown of the AKT3 (PKB $\gamma)$, PI3KCA, and VEGFR2 genes by RNA interference suppresses glioblastoma multiforme T98G cells invasiveness in vitro. Tumour Biol 36: 3263-3277, 2015.

36. Höland K, Boller D, Hagel C, Dolski S, Treszl A, Pardo OE, Cwiek P, Salm F, Leni Z, Shepherd PR, et al: Targeting class IA PI3K isoforms selectively impairs cell growth, survival, and migration in glioblastoma. PLoS One 9: e94132, 2014.

37. Broadley KW, Hunn MK, Farrand KJ, Price KM, Grasso C, Miller RJ, Hermans IF and McConnell MJ: Side population is not necessary or sufficient for a cancer stem cell phenotype in glioblastoma multiforme. Stem Cells 29: 452-461, 2011. 
38. Jamieson S, Flanagan JU, Kolekar S, Buchanan C, Kendall JD, Lee WJ, Rewcastle GW, Denny WA, Singh R, Dickson J, et al: A drug targeting only p1 $10 \alpha$ can block phosphoinositide 3-kinase signalling and tumour growth in certain cell types. Biochem $\mathrm{J}$ 438: 53-62, 2011.

39. Jackson SP, Schoenwaelder SM, Goncalves I, Nesbitt WS, Yap CL, Wright CE, Kenche V, Anderson KE, Dopheide SM, Yuan Y, et al: PI 3-kinase p110beta: A new target for antithrombotic therapy. Nat Med 11: 507-514, 2005.

40. Sadhu C, Masinovsky B, Dick K, Sowell CG and Staunton DE: Essential role of phosphoinositide 3-kinase delta in neutrophil directional movement. J Immunol 170: 2647-2654, 2003.

41. Maira SM, Stauffer F, Brueggen J, Furet P, Schnell C, Fritsch C, Brachmann $\mathrm{S}$, Chène $\mathrm{P}$, De Pover A, Schoemaker K, et al: Identification and characterization of NVP-BEZ235, a new orally available dual phosphatidylinositol 3-kinase/mammalian target of rapamycin inhibitor with potent in vivo antitumor activity. Mol Cancer Ther 7: 1851-1863, 2008.

42. Berridge MV, Herst PM and Tan AS: Tetrazolium dyes as tools in cell biology: New insights into their cellular reduction. Biotechnol Annu Rev 11: 127-152, 2005.

43. Berridge MV and Tan AS: Characterization of the cellular reduction of 3-(4,5-dimethylthiazol-2-yl)-2,5-diphenyltetrazolium bromide (MTT): Subcellular localization, substrate dependence, and involvement of mitochondrial electron transport in MTT reduction. Arch Biochem Biophys 303: 474-482, 1993

44. Fan QW, Cheng CK, Nicolaides TP, Hackett CS, Knight ZA, Shokat KM and Weiss WA: A dual phosphoinositide-3-kinase alpha/mTOR inhibitor cooperates with blockade of epidermal growth factor receptor in PTEN-mutant glioma. Cancer Res 67: 7960-7965, 2007.

45. He K, Xu T, Xu Y, Ring A, Kahn M and Goldkorn A: Cancer cells acquire a drug resistant, highly tumorigenic, cancer stem-like phenotype through modulation of the PI3K/Akt/ $\beta$-catenin/CBP pathway. Int J Cancer 134: 43-54, 2014

46. Matsubara S, Ding Q, Miyazaki Y, Kuwahata T, Tsukasa K and Takao S: mTOR plays critical roles in pancreatic cancer stem cells through specific and stemness-related functions. Sci Rep 3: 3230, 2013

47. Kingham $\mathrm{E}$ and Welham M: Distinct roles for isoforms of the catalytic subunit of class-IA PI3K in the regulation of behaviour of murine embryonic stem cells. J Cell Sci 122: 2311-2321, 2009.
48. Lathia JD, Mack SC, Mulkearns-Hubert EE, Valentim CL and Rich JN: Cancer stem cells in glioblastoma. Genes Dev 29: 1203-1217, 2015.

49. Zhang R, Banik NL and Ray SK: Differential sensitivity of human glioblastoma LN18 (PTEN-positive) and A172 (PTENnegative) cells to Taxol for apoptosis. Brain Res 1239: 216-225, 2008.

50. Meier TI, Cook JA, Thomas JE, Radding JA, Horn C, Lingaraj T and Smith MC: Cloning, expression, purification, and characterization of the human class Ia phosphoinositide 3-kinase isoforms Protein Expr Purif 35: 218-224, 2004.

51. Beeton CA, Chance EM, Foukas LC and Shepherd PR: Comparison of the kinetic properties of the lipid- and proteinkinase activities of the p110alpha and p110beta catalytic subunits of class-Ia phosphoinositide 3-kinases. Biochem J 350: 353-359, 2000.

52. Schwartz S, Wongvipat J, Trigwell CB, Hancox U, Carver BS, Rodrik-Outmezguine V, Will M, Yellen P, de Stanchina E, Baselga $\mathrm{J}$, et al: Feedback suppression of PI3Ka signaling in PTEN-mutated tumors is relieved by selective inhibition of PI3K $\beta$. Cancer Cell 27: 109-122, 2015.

53. Costa C, Ebi H, Martini M, Beausoleil SA, Faber AC, Jakubik CT, Huang A, Wang Y, Nishtala M, Hall B, et al: Measurement of PIP3 levels reveals an unexpected role for p110 $\beta$ in early adaptive responses to $\mathrm{p} 110 \alpha$-specific inhibitors in luminal breast cancer. Cancer Cell 27: 97-108, 2015.

54. Sunayama J, Matsuda K, Sato A, Tachibana K, Suzuki K, Narita Y, Shibui S, Sakurada K, Kayama T, Tomiyama A, et al: Crosstalk between the PI3K/mTOR and MEK/ERK pathways involved in the maintenance of self-renewal and tumorigenicity of glioblastoma stem-like cells. Stem Cells 28: 1930-1939, 2010.

55. Gough DJ, Koetz L and Levy DE: The MEK-ERK pathway is necessary for serine phosphorylation of mitochondrial STAT3 and Ras-mediated transformation. PLoS One 8: e83395, 2013.

56. Soares HP, Ming M, Mellon M, Young SH, Han L, Sinnet-Smith J and Rozengurt E: Dual PI3K/mTOR inhibitors induce rapid overactivation of the MEK/ERK pathway in human pancreatic cancer cells through suppression of mTORC2. Mol Cancer Ther 14: 1014-1023, 2015 .

57. Toulany M, Minjgee M, Saki M, Holler M, Meier F, Eicheler W and Rodemann HP: ERK2-dependent reactivation of Akt mediates the limited response of tumor cells with constitutive K-RAS activity to PI3K inhibition. Cancer Biol Ther 15: 317-328, 2014. 